\title{
Towards Computer-Assisted Deep Brain Stimulation Targeting with Multiple Active Contacts
}

\author{
Silvain Bériault, Yiming Xiao, Lara Bailey, D. Louis Collins, \\ Abbas F. Sadikot, and G. Bruce Pike \\ McConnell Brain Imaging Centre, Montreal Neurological Institute, \\ 3801 University Street, Montreal, Quebec, H3A 2B4, Canada \\ silvain.beriault@mail.mcgill.ca
}

\begin{abstract}
We present a novel method for preoperative computer-assisted deep brain stimulation (DBS) electrode targeting that takes into account the multiplicity of available contacts and their polarity. Our framework automatically evaluates the efficacy of many possible electrode orientations to optimize the interplay between the extracellular electric field, created from distinct arrangements of active contacts, and anatomical structures responsible for therapeutic and potential side effects. Experimental results on subthalamic DBS cases suggest bipolar configurations provide more flexibility and control on the spread of electric field and, consequently, are most robust to targeting imprecision. Visualization of predicted efficacy maps provides surgeons with complementary feedback that can bridge the gap between insertion safety and optimal therapeutic efficacy. Overall, this work adds a new dimension to preoperative DBS planning and suggests new insights regarding multi-target stimulation.
\end{abstract}

Keywords: Deep brain stimulation, electric field modeling, Parkinson's disease, histological atlas, image-guided neurosurgery.

\section{Introduction}

Deep brain stimulation (DBS) is an increasingly important neurosurgical treatment for severe pharmacologically-resistant Parkinson's disease (PD), and other movement and affective disorders. The procedure involves the implantation of multi-contact stimulating electrodes in deep brain structures via minimally invasive image-guided neurosurgery (IGNS). Effective neuromodulation is highly dependent upon precise electrode targeting and programming to simultaneously maximize symptom relief and minimize side effects caused by stimulation of neighbouring anatomical structures. For example, bilateral high frequency DBS of the subthalamic nuclei (STN) is effective to reduce motor fluctuations, dyskinesias, rigidity, tremor and slowness of movement symptoms that characterize advanced PD. However, targeting inaccuracies can yield side effects due to the spread of extracellular electric field to the internal capsule (muscle contraction), the occulomotor nerve root (diplopia), the superior cerebellar peduncle (ataxia), the medial lemniscus and spinothalamic pathways (paresthesias) [1]. 
The most common clinical protocol for DBS targeting is carried in two phases. $B e$ fore the operation, the neurosurgeon approximates the target position on the patient's preoperative MRI. However, typical DBS target sites, such as the STN, do not possess clear boundaries on clinical MRIs and the surgeon must often rely on the relative position of other anatomical landmarks (e.g. the AC-PC line) or on a stereotactic atlas of the basal ganglia that is deformed to the patient's datasets [2]. During the operation, micro-electrode recording (MER) of neuronal activity is used to refine the physiological extent of the targeted nucleus and neighbouring nuclei. Furthermore, monopolar (single contact) micro- and macro-stimulation is performed to determine salutary and untoward effects of stimulation.

Recently, several computer-assisted tools were developed to help neurosurgical teams with different aspects of the overall procedure. Some software platforms (e.g. [3-4]) were proposed to allow fusion of multi-modal MRI, automatic segmentation of key anatomical structures, registration to anatomical and functional atlases, and effective visualization of DBS lead trajectory. Advanced MRI techniques have also developed for direct visualization of the STN and other basal ganglia structures [5-6]. Probabilistic functional atlases, relating target sites to clinical outcome, have been used for semi-automatic or automatic target prediction [7-8]. Algorithms were also developed for automatic trajectory optimization to minimize hemorrhagic risks and loss of function (e.g. [9-10]).

However, current planning methods for preventing electric field propagation into neighbouring structures without compromising treatment efficacy are still performed intraoperatively, once the burr-hole has been made. An isotropic Gaussian model is often used to visualize the extent of cathodal (monopolar) stimulation and to build multi-subject probabilistic efficacy atlases [7-8]. A spherical shell kernel [11] was also proposed with the assumption that therapeutic effects arise from stimulating specific tissue located on an annulus, around the electrode, rather than the total volume of tissue activated. Finally, iterative finite element models (FEM) [12] have been proposed to account for the contact geometry and material, and anisotropic white matter conductivity, based diffusion tensor imaging (DTI). This latter approach is particularly useful for a posteriori optimization of the programming parameters given a final electrode position. However, the model is limited by the low spatial resolution of DTI datasets (typically $2 \times 2 \times 2-\mathrm{mm}$ ) and is computationally expensive for a priori planning.

This work presents a novel computer-assisted planning framework for a priori electrode targeting. A map of anatomical regions responsible for therapeutic and potential side effects is derived from a high-resolution basal ganglia histology atlas nonlinearly registered to patient's $\mathrm{T} 1 \mathrm{w}$ and susceptibility-based $\mathrm{T} 2 * \mathrm{w}$ datasets acquired within a clinically acceptable time via a multi-echo MRI sequence. A fast practical model of the extracellular electric field is proposed to predict the spread into the target and neighbouring structures at increasing stimulation intensities, and to compute an overall efficacy measure for different arrangements of active contacts and polarities. Overall, we developed a new software simulation that mimics microand macro-stimulation preoperatively allowing many electrode orientations, depths and multi-contact configurations to be evaluated before entering the operating room. 


\section{$2 \quad$ Method}

Our optimization framework relies on the interplay between the electric field generated from the stimulation of one or multiple active contacts and specific brain areas responsible for therapeutic and side effects. Section 2.1 describes the MRI acquisition protocol and registration to a high-resolution histology-derived atlas. Section 2.2 describes the analytical model used to describe the electric field and section 2.3 presents the novel planning and optimization algorithm.

\subsection{MRI Acquisition and Image Processing}

Multi-contrast MRI acquisition of the entire head is obtained from a 3D gradient echo MRI sequence with $0.95 \mathrm{~mm}$ isotropic resolution and 10 echoes $(\mathrm{TR}=30 \mathrm{~ms}$, $\left.\mathrm{TEs}=\{1.6 ; 4.1 ; 6.6 ; 9.1 ; 13.0 ; 16.0 ; 18.5 ; 21.0 ; 23.5 ; 26.0\} \mathrm{ms}, \alpha=23^{\circ}, \mathrm{BW}=450 \mathrm{~Hz}\right)$. The magnitude images from the first four echoes are averaged to provide suitable $\mathrm{T} 1 \mathrm{w}$ contrast for neuronavigation. The magnitude images from the last five echoes are averaged to provide $\mathrm{T} 2 * \mathrm{w}$ contrast and direct imaging of the STN. The total acquisition time is 7:05 min on a 3T Siemens TIM Trio scanner and a 32-channel coil. The $\mathrm{T} 1 \mathrm{w}$ and $\mathrm{T} 2 * \mathrm{w}$ datasets are intrinsically co-registered as they originate from the same acquisition.

A map of side effect areas is obtained from non-linear registration of the patient's T1w MRI with an existing high resolution $(0.034 \times 0.034 \times 0.70-\mathrm{mm})$ histological atlas of the basal ganglia and thalamus (see Fig. 1a), using the Colin27 T1w average template as an intermediate volume [13]. For this prototype, the potential side effect regions were defined as the internal capsule, the superior cerebellar peduncle and the medial lemniscus pathways. To improve the registration accuracy of the small-size STN (not visible on T1w contrast), we used a similar non-linear registration method that integrates multiple MRI contrasts (T1w and T2*w) and an intensity inverted T2w Colin template as an intermediate volume (see [14] for implementation details and validation). The raw T1w and T2*w datasets are shown in Fig. 1b-c. Fig. 1d shows STN labeling over the $\mathrm{T} 2 * \mathrm{w}$ dataset. Fig. 1e shows the final therapeutic and potential side effect regions overlaid on the $\mathrm{T} 1 \mathrm{w}$ dataset.
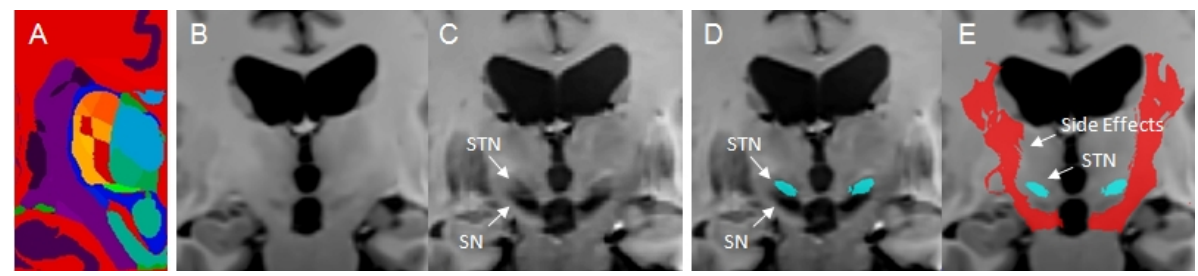

Fig. 1. (a) High resolution basal ganglia atlas (left side). (b) The T1w navigation dataset. (c) The $\mathrm{T} 2 * \mathrm{w}$ dataset exhibiting STN and substantia nigra (SN) contrast. (d) Atlas-based warping of the STN label overlaid on the $\mathrm{T} 2{ }^{*} \mathrm{w}$ dataset. (e) Final therapeutic and side effects regions overlaid on the $\mathrm{T} 1 \mathrm{w}$ navigation dataset. 


\subsection{Modeling the DBS Electric Field}

Recently, Zhang and Grill [15] demonstrated that a point source model and a homogeneous brain tissue conductivity assumption yield comparable electric field and volume of tissue activated in comparison to a full FEM for small-sized contacts. This key observation enables the design of fast and representative analytical approximations of the extracellular electric field. Thus, for a point source delivering current $I_{0}$ (A), the current density $\vec{J}(r)\left(\mathrm{A} / \mathrm{m}^{2}\right)$ on a spherical surface of radius $r(\mathrm{~m})$ is given as the current divided by the surface area: $\vec{J}(r)=I_{0} / 4 \pi r^{2}$. From the generalized Ohm law $(\vec{J}=\sigma \vec{E})$ where $\sigma$ is the tissue conductance $(\mathrm{S} / \mathrm{m})$ and $\vec{E}$ the electric field (V/m), or the negative gradient of the extracellular potential $(\vec{E}=-\nabla V)$, the electric potential $(V)$ at a distance $r$ of the source is obtained from the following surface integration:

$$
\int_{0}^{r}-\nabla V d r=\int_{0}^{r} \frac{-I_{0}}{4 \pi \sigma r^{2}} d r, \text { and } V=\frac{I_{0}}{4 \pi \sigma r}
$$

Eq. (1) describes the special case of single contact (monopolar) stimulation. Bipolar and other multi-contact configurations, often required for STN DBS [1] due to the proximity to the internal capsule, can be obtained using superposition. For example, the potential for bipolar stimulation becomes:

$$
V=\frac{I_{0}}{4 \pi \sigma}\left(\frac{1}{r_{1}}-\frac{1}{r_{2}}\right)
$$

In Eq. (2), $r_{1}$ is the distance from the cathodal contact and $r_{2}$ the distance from the anodal contact. A similar expression is obtained for tripolar stimulation (not shown). Hence, monopolar stimulation is a special case of multi-contact DBS with $r_{2}=\infty$. Fig. 2 shows the potential and electric field distributions for monopolar and bipolar cases (with $I_{0}=4 \mathrm{~mA}$ and $\sigma=0.2 \mathrm{~S} / \mathrm{m}$ ). The main advantage of bipolar stimulation is that the electrical field strength decays more rapidly, thus reducing the risk of stimulating neighbouring structures.
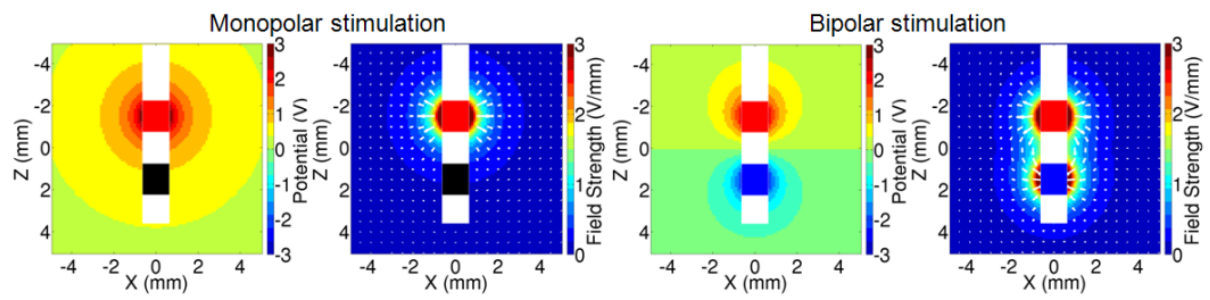

Fig. 2. Extracellular potential and electric field using point source contacts with $I_{0}=4 \mathrm{~mA}$, $\sigma=0.2 \mathrm{~S} / \mathrm{m}$. For the monopolar case, lines of electric forces arising from the cathodal contact radiates isotropically. For the bipolar case, lines of electric forces are partially attracted to the anodal contact. 


\subsection{Preoperative Electrode Targeting Optimization}

Using the therapeutic and side effects maps and the electric field model described in sections 2.1 and 2.2, we developed a software simulation that mimics micro- and macro-stimulation to evaluate many possible electrode orientations, depths and configurations preoperatively. To do so, we define an efficacy likelihood measure $(E F)$ that modulates the interplay between a therapeutic effect profile $\left(P_{T E}\right)$ and a side effect profile $\left(P_{S E}\right)$. Fig. 3 shows the theoretical behavior of $P_{T E}$ and $P_{S E}$. At low intensity $(I)$, the likelihood of therapeutic and side effects is low. As the stimulation intensity increases, both $P_{T E}$ and $P_{S E}$ increase, because the stronger electric field will spread into more brain tissue. However, $P_{T E}$ will typically increase faster then $P_{S E}$, because the electrode, unless off-target, is closer to the therapeutic areas than the side effects areas and will stabilize once the small-sized targeted nucleus is completely stimulated. Depending on the targeting accuracy, both $P_{T E}$ and $P_{S E}$ can be shifted to the left or right and our goal is to maximize the $E F$ measure:

$$
E F=\int_{0}^{I_{\max }} P_{T E}(I) d I-\int_{0}^{I_{\max }} P_{S E}(I) d I
$$

For a given electrode orientation, depth, configuration of active contacts, and stimulation intensity, $P_{T E}(I)$ and $P_{S E}(I)$ relates to the sum of a voxel-wise activation likelihood $\left(\mathrm{P}_{\mathrm{A}}\right)$ measured at specific voxels responsible for therapeutic and side effects:

$$
P_{A}(x, y, z)=\left\{\begin{array}{cc}
1 & E(x, y, z)>E_{\max } \\
E(x, y, z) / E_{\max } & E(x, y, z)<E_{\max }
\end{array}\right.
$$

In Eq. (4), $E_{\max }$ is a user-defined parameter corresponding to electric field strength where activation is maximal $\left(P_{A}=1.0\right)$ with a $1: 4$ ratio for anodal contacts because anodic stimulation thresholds are consistently higher than cathodic thresholds [15]. Finally, $P_{T E}$ and $P_{S E}$ profiles are computed at increasing intensity $(I)$ and the efficacy $(E F)$ is computed via a discrete implementation of Eq. (3). The depth at which $E F$ is maximal is kept for each evaluated electrode orientation and configuration.

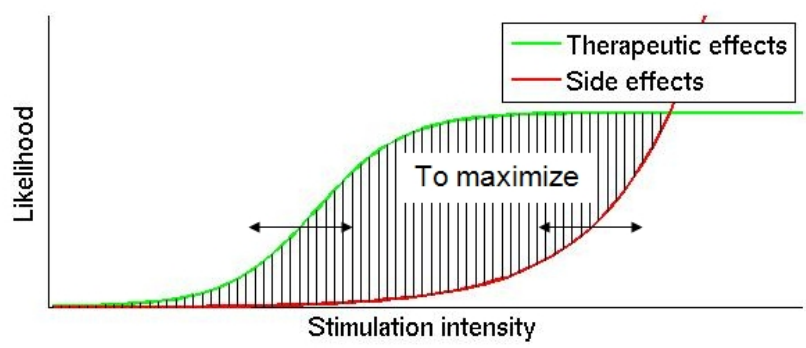

Fig. 3. Theoretical plot showing the interplay between the likelihood of therapeutic $\left(P_{T E}\right)$ and possible side effects $\left(P_{S E}\right)$ as the stimulation intensity increases. The goal is to maximize the efficacy $(E F)$, which is modeled as the area between $P_{T E}$ and $P_{S E}$ curves and which can decrease and even become negative when the electrode is outside the targeted structure. 


\section{$3 \quad$ Results}

A preliminary evaluation was performed on 3 PD patients (STN DBS). For each case, an initial search space of possible electrode orientations was defined from the identification of a tentative target point, within the dorsolateral STN (motor part) on the $\mathrm{T} 2 * \mathrm{w}$ dataset, and a set of possible brain entry points, within the frontal lobe because it corresponds to typical insertion area for STN DBS. The search space was then automatically filtered [10] to exclude orientations yielding lead trajectories that approach sulci, ventricles and blood vessels at an unsafe distance. The remaining orientations were analyzed with our targeting optimization method and using the Medtronic 3389 electrode model (diameter: $1.27 \mathrm{~mm}$, contact height: $1.5 \mathrm{~mm}$, contact spacing: $0.5 \mathrm{~mm}$ ). Fig. 4 displays a color-coded map of the predicted efficacy, relatively to other orientations, for monopolar, wide bipolar and narrow bipolar. Fig. 5 plots the electric field at the predicted electrode depth for a specific orientation (subject 1). For all configurations, the cathodal contact responsible for most of tissue activation is well positioned within the dorsolateral STN.

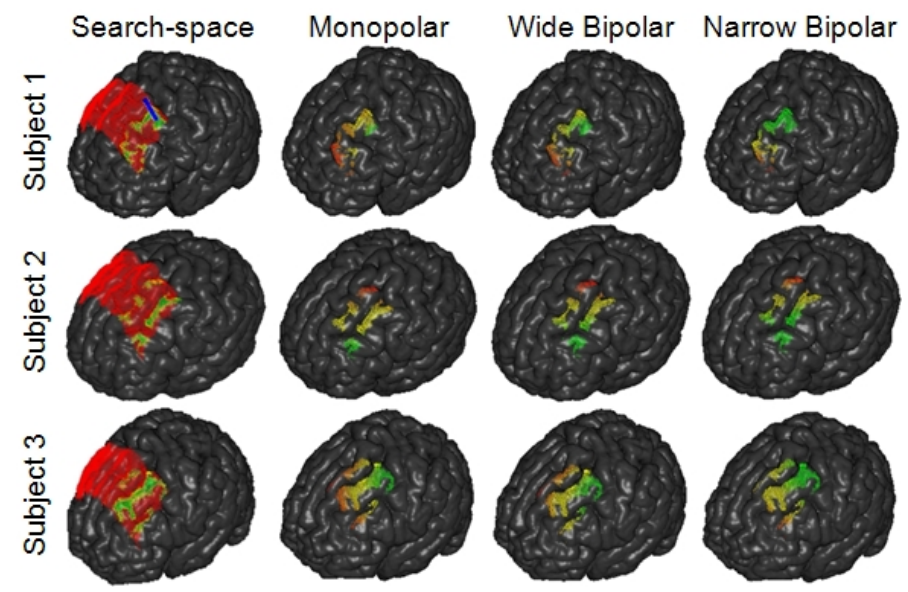

Fig. 4. Given an initial search space, our framework computes and allows immediate visualization of the computed efficacy for the different orientations and configurations of active contacts. Color scale for search-space column: green=safe, yellow=acceptable: red=rejected trajectories. Color scale for other columns: green=high, yellow=average, red=low predicted efficacy

Although more efficient in terms of battery consumption, monopolar STN DBS with optimal efficacy is concentrated onto a smaller patch of entry points (orientations) in comparison to the bipolar configurations (see Fig. 4). Furthermore, the narrow bipolar configuration provides the most control over to the spread of electric field into neighbouring structures, especially at high stimulation intensity, due to the small distance separating the cathodal and anodal contacts (see Fig. 5). Thus, this configuration is most robust to targeting imprecision. Interestingly, our software predicted anterior approaches for subject 2 and posterior approaches for subjects 1 and 3 yield 
superior efficacy. This can be attributed to the use of patient-specific search spaces, which take into account the high inter-subject variability of gyral patterns and ventricular size.
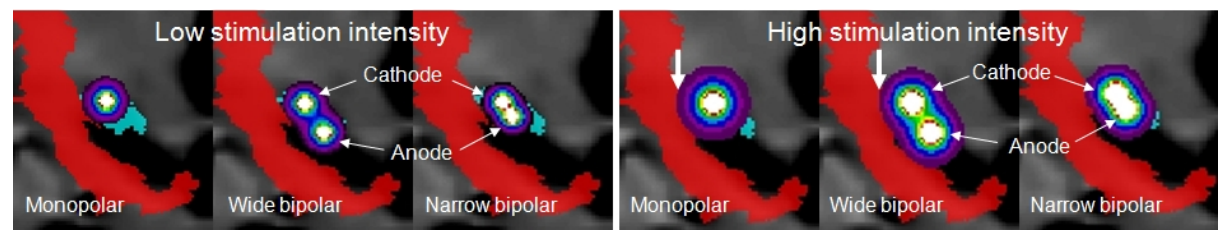

Fig. 5. Coronal view of the electric field for subject 1 on a specific electrode orientation (see blue marker on Fig. 4) at predicted depth for different configurations. At low stimulation intensity $(4 \mathrm{~mA})$, any configuration can be used without propagation to the internal capsule. At high stimulation $(10 \mathrm{~mA})$, the electric field overlaps with the internal capsule at the cathodal contact of both monopolar and wide bipolar configuration, hence increasing the risk of side effects.

\section{Discussion and Conclusions}

We presented a new electrode targeting optimization method that takes into account the multiplicity of available contacts on standard DBS lead. To our knowledge, this is the first published method that automatically computes an efficacy measure, for several possible electrode orientations, depths and configurations, based on the interplay between the created electric field and patient-specific maps of therapeutic and possible side effects. Within the context of automatic DBS targeting we also proposed the novel integration of a high-resolution basal ganglia histology atlas non-linearly registered to multi-contrast patient's datasets (T1w and $\mathrm{T} 2 * \mathrm{w})$ simultaneously acquired using a multi-echo MRI sequence. Our preliminary results reveal that our method provides the surgeon with high-level feedback, which is complementary to other planning considerations, and that can bridge the gap between lead insertion safety and optimal therapeutic efficacy.

Our modular framework also allows the use of alternate techniques for creating maps of therapeutic and possible side effects. Indeed, the small-sized STN could be segmented manually from the $\mathrm{T} 2 * \mathrm{w}$ dataset, thus eliminating possible atlas registration inaccuracies. Otherwise, functional atlases constructed from a population of previous DBS surgeries could have also been used. However, recent advances in structural MRI pave the way to custom solutions based on the patient specific anatomy, and also enable simulated exploration of other anatomical targets and alternate DBS insertion strategies. Overall, the proposed software adds a new dimension to preoperative DBS planning and suggests new insights regarding multi-contact and multi-target stimulation, which may reveal essential as DBS becomes applicable to a wider variety of diseases and symptoms and with the increasing number of available contacts on new generations of lead models. For future work, we will calibrate the proposed therapeutic and side effect profiles using intraoperative micro- and macrostimulation recordings and we will incorporate a more accurate electrothermal model that takes into account white matter fiber orientation and presence of blood vessels via patient-specific DTI and SWI venography datasets. 


\section{References}

1. Montgomery, E.B.: Deep Brain Stimulation Programming: Principles and Practice. Oxford University Press (2010)

2. Brunenberg, E.J., Platel, B., Hofman, P.A., Ter Haar Romeny, B.M., Visser-Vandewalle, V.: Magnetic resonance imaging techniques for visualization of the subthalamic nucleus. J. Neurosurg. 115, 971-984 (2011)

3. Nowinski, W.L., Yang, G.L., Yeo, T.T.: Computer-aided stereotactic functional neurosurgery enhanced by the use of the multiple brain atlas database. IEEE Trans. Med. Imaging 19, 62-69 (2000)

4. D'Haese, P.F., Pallavaram, S., Li, R., Remple, M.S., Kao, C., Neimat, J.S., Konrad, P.E., Dawant, B.M.: CranialVault and its CRAVE tools: A clinical computer assistance system for deep brain stimulation (DBS) therapy. Med. Image Anal. 16, 744-753 (2012)

5. Elolf, E., Bockermann, V., Gringel, T., Knauth, M., Dechent, P., Helms, G.: Improved visibility of the subthalamic nucleus on high-resolution stereotactic MR imaging by added susceptibility $(\mathrm{T} 2 *)$ contrast using multiple gradient echoes. Am. J. Neuroradiol. 28, 10931094 (2007)

6. Xiao, Y., Beriault, S., Pike, G.B., Collins, D.L.: Multi-contrast multi-echo FLASH MRI for targeting the subthalamic nucleus. Magn. Reson. Imaging 30, 627-640 (2012)

7. D'Haese, P.F., Cetinkaya, E., Konrad, P.E., Kao, C., Dawant, B.M.: Computer-aided placement of deep brain stimulators: from planning to intraoperative guidance. IEEE Trans. Med. Imaging 24, 1469-1478 (2005)

8. Guo, T., Parrent, A.G., Peters, T.M.: Surgical targeting accuracy analysis of six methods for subthalamic nucleus deep brain stimulation. Comput. Aided Surg. 12, 325-334 (2007)

9. Essert, C., Haegelen, C., Lalys, F., Abadie, A., Jannin, P.: Automatic computation of electrode trajectories for Deep Brain Stimulation: a hybrid symbolic and numerical approach. Int. J. Comput. Assist. Radiol. Surg. (2011)

10. Bériault, S., Al Subaie, F., Mok, K., Sadikot, A.F., Pike, G.B.: Automatic Trajectory Planning of DBS Neurosurgery from Multi-modal MRI Datasets. In: Fichtinger, G., Martel, A., Peters, T. (eds.) MICCAI 2011, Part I. LNCS, vol. 6891, pp. 259-266. Springer, Heidelberg (2011)

11. Pallavaram, S., D’Haese, P.-F., Kao, C., Yu, H., Remple, M., Neimat, J., Konrad, P., Dawant, B.: A New Method for Creating Electrophysiological Maps for DBS Surgery and Their Application to Surgical Guidance. In: Metaxas, D., Axel, L., Fichtinger, G., Székely, G. (eds.) MICCAI 2008, Part I. LNCS, vol. 5241, pp. 670-677. Springer, Heidelberg (2008)

12. McIntyre, C.C., Mori, S., Sherman, D.L., Thakor, N.V., Vitek, J.L.: Electric field and stimulating influence generated by deep brain stimulation of the subthalamic nucleus. Clin. Neurophysiol. 115, 589-595 (2004)

13. Chakravarty, M.M., Bertrand, G., Hodge, C.P., Sadikot, A.F., Collins, D.L.: The creation of a brain atlas for image guided neurosurgery using serial histological data. Neuroimage 30, 359-376 (2006)

14. Xiao, Y., Bailey, L., Mallar Chakravarty, M., Beriault, S., Sadikot, A.F., Pike, G.B., Collins, D.L.: Atlas-Based Segmentation of the Subthalamic Nucleus, Red Nucleus, and Substantia Nigra for Deep Brain Stimulation by Incorporating Multiple MRI Contrasts. In: Abolmaesumi, P., Joskowicz, L., Navab, N., Jannin, P. (eds.) IPCAI 2012. LNCS, vol. 7330, pp. 135-145. Springer, Heidelberg (2012)

15. Zhang, T.C., Grill, W.M.: Modeling deep brain stimulation: point source approximation versus realistic representation of the electrode. J. Neural. Eng. 7, 1-11 (2010) 